\title{
Building Shared Guardianship through Blockchain Technology and Digital Museum Objects
}

\author{
Frances Liddell
}

\begin{abstract}
This article considers the notion of shared guardianship in the context of digital museum objects and blockchain technology and it argues that the technology can contribute to the production of value in digital museum objects that goes beyond the monetary. Shared guardianship is understood to be a process of prioritising the experience of others and forming a diverse set of stakeholders that transforms understandings around ownership, meanwhile, a blockchain is a type of distributed ledger technology which can be used to identify digital files and so create digital files that feel ownable and authentic. As such, this paper argues that blockchain technology could create a new layer of materiality and value in digital museum objects which could support the formation of shared guardianship. This question will be analysed in relation to the theoretical underpinnings of digital materiality and a case study project at the National Museums Liverpool in Liverpool, UK, which investigated how to implement blockchain technology in the museum context in order to produce collective ownership and meaningful, connected digital objects.
\end{abstract}

Key words: Value, Ownership, Digital Materiality, Shared Guardianship, Authenticity, NFTs.

\section{Introduction}

This paper focuses on the notion of guardianship in the context of the digital museum object. We can consider guardianship as a process of defending or protecting; for example, a museum is often described as a guardian of its collection as it both preserves and protects the cultural property contained within its walls. In this way, the concept is embedded into the notion of ownership, and, in her discussion of the term and cultural property, Haidy Geismar (2008) argues that guardianship is not only part of this concept but it also transforms our understanding of ownership as it encourages a more dynamic and collaborative relationship to form. Hence, guardianship is not simply about protecting or preserving, it also involves the forging of new and ongoing relations. Janet Marstine $(2011,2017)$ also considers this idea under the term of shared guardianship. She argues that culture is relative and shared and that privileging experience can forge new relationships between people and objects, thus the process helps in 'binding people together through things' (Marstine 2017: 46).

In building on this notion of shared guardianship, the following discussion will consider to what extent guardianship can be formed over the digital museum object and, in so doing, will produce digital objects which feel both valued and meaningful. Here, meaningful refers to producing objects which have a personal value; they are not necessarily valuable in a monetary sense but rather for the connection they represent. A digital museum object refers to digital images displayed in a museum's digital collection and this discussion takes the perspective that, as images presented through the Internet, these images are viewed as open, reproducible and distributed (Kallinikos et al. 2010; Jeffrey 2015). These attributes bring about a set of challenges when considering the formation of shared guardianship as they assume that digital images are viewed as less valuable than physical objects, because it is difficult to form exclusivity around them (Jeffrey 2015; Atasoy and Morewedge 2018). In this way, these attributes challenge the ability to claim an ownership over digital images. 
This problem will be examined in the context of a case study at the National Museums Liverpool (NML) in Liverpool, UK, which investigates how to implement blockchain technology in the museum context so as to produce collective ownership and meaningful, connected digital objects. Blockchain is a type of distributed ledger technology which encourages a way of creating value in the digital space by singularizing digital tokens through recording these entities onto the distributed ledger. This enables these tokens to be claimed as owned and have a proven authenticity, as this process of documentation produces an immutable provenance. Thus, the technology's digital infrastructure builds in authenticity and exclusivity for any token exchanged within the blockchain network, and this suggests that blockchain's materiality is different from that of a 'typical' or non-blockchained digital object. In this circumstance, materiality denotes more than physical presence. In looking to literature on the concept of digital materiality, Matthew Kirschenbaum (2008) identifies forensic and formal materiality as two aspects to this concept. These forms focus on the individualization of the bits and bytes and, unlike atoms, Kirschenbaum (2008) argues that these bits and bytes are weightless and symbolic; however, they still leave traces which provide evidence of their materiality. On the other hand, Johanna Drucker (2013) considers digital materiality from a performative perspective and argues for a need to look at what materials do rather than simply what they are. Drucker (2013) also argues for a distributed perspective of materiality which highlights the different actors needed for a digital object to exist. Therefore, there are layers to the term 'digital materiality' and section three will use these diverging aspects as a framework in which to analyze how blockchain's materiality can build value in digital tokens exchanged in its network.

In contrast, section two will review literature on the understanding of value in the digital museum object and, building on the discussions in both sections two and three, section four will examine the case study at the NML and argue that digital museum objects could play a role in the building of relations through shared guardianship. The NML is a collection of seven museums located in and around Liverpool, UK, compromising of the Walker Art Gallery, the World Museum, the Museum of Liverpool, Sudley House, the Maritime Museum, the International Slavery Museum, and the Lady Lever Art Gallery. This project took place primarily at the Walker Art Gallery, the World Museum and on the NML website. The project used a collaborative approach to explore the museum collections, which privileged the experience of the participant by documenting their relationship about an object to a digital and blockchain-enabled version of the object. In so doing, the participants claimed ownership over a digital token of the museum's collection and this ownership could be proven using blockchain technology. In developing the findings of this project, this paper argues that reconceptualizing ownership in the context of digital museum collections has the potential to forge relations between the viewer, object and museum, bringing about a sense of shared ownership or guardianship towards the digital museum object. As such, this process also redefines the role of the digital museum object and produces digital objects which are not only ownable but also feel meaningful. However, before considering these arguments, the following section will examine the key concepts in the context of museological literature.

\section{Meaningful Digital Museum Objects}

In museums, there is often a perception that objects are made meaningful through the curatorial expert (Srinivasan et al. 2010), and this process produces an authority in which the museum is viewed as the 'unassailable voice' of its collections (Walsh 1997). Susan Hazan highlights how this understanding leads to museums being viewed as 'floating above the community' (Hazan 2007: 136). In other words, the museum is prioritized as the expert in knowledge above any communities, including source communities and the audiences it serves, and it is this expertise that renders objects in its collections as significant.

However, this perception about museums and their authority has been challenged in museological literature and practice, notably, but not exclusively, with the concept of the 'postmuseum', which identifies a paradigm shift where the museum focuses on being more public and inclusive (Hooper-Greenhill 2000b). Such a shift involves a more two-way communication in the interpretation of collections which encourages viewers to play an active role in looking 
at, and responding to, the object (Hooper-Greenhill 2000a). We can see this idea in poststructuralism and reception theory where objects are viewed as polysemic in meaning, as knowledge obtained from them is contingent on the viewer rather than just its material and a single expert (Hooper-Greenhill 2000b; Marstine 2017).

By engaging in these processes, museums can produce narratives in their collections which are more relevant and meaningful to the audiences and communities they serve (Merriman 2015) and, indeed, it can even dilute the authority of a museum as it takes a collaborative approach (Cameron and Robinson 2007). Museum objects also become a point of discussion rather than signify one singular historic narrative and this approach encourages participants to invest into this process, which, in so doing, grows the potential for shared guardianship to form. As noted in the introduction, shared guardianship focuses on prioritizing the experience of others, transforming the notion of ownership and creating a diverse set of stakeholders (Geismar 2008; Marstine 2011, 2017). Hence, shared guardianship aims to strengthen ties to communities through the process of collaboration and investment.

The digital offers a different perspective in this discussion. For example, Nicole Meehan (2020) proposes that the digital object could act as a meeting point, in which the digital surrogate of an artefact captures the different memories and knowledge associated with the physical artefact. The exhibition Tales of a Changing Nation at the National Museum of Scotland in 2011 offers a case in point as visitors were invited to interact with QR codes displayed by different museum artefacts in the exhibit and could access digital media footage and digital 'living memories' of these artefacts presented by other visitors (Speed 2012). These memories consisted of a form of oral history as visitors could document their responses to the artefact in a digital format and these were available to others who passed through the exhibition and interacted with the $Q R$ code. As a result, the digital version of the artefact acted as a meeting point of different memories and information about the physical artefact in the exhibition, and it also opened up the opportunity for visitors to be part of the construction of knowledge about that particular artefact.

This example suggests the digital museum object could be used as a relational device where visitors could invest their own experience into the discussion about the object, thus making the object more meaningful and valued. This understanding resonates with the conclusions of Stuart Jeffrey, Siân Jones, Mhairi Maxwell, Alex Hale \& Cara Jones (2020), who highlight how the process of co-designing digital recordings of heritages sites with communities produces new forms of value and significance, as well as challenge existing attributed value. As such, the process of collaboration both moulds understanding and creates digital surrogates that reflect the perspectives of the participants. Of course, this approach to digital museum objects faces that challenge that museum digital objects are assumed to be less 'valuable' than physical objects because of their perceived lack of materiality and their open and distributed structure (Kallinikos et al. 2010; Jeffrey 2015; Atasoy and Morewedge 2018). However, in cases such as the digitization of Maori taonga (translated as "cultural treasures') this assumption does not appear to be evident and rather, value is formed through the social relations and knowledge initiated through new digital tools and surrogates (Brown 2007; Ngata et al. 2012; Geismar 2018). In other words, different cultures understand and acknowledge value of digital artefacts to varying degrees.

Other research suggests that the digital museum object could be used as a form of digital contact zone (Hogsden and Poulter 2012). Here, the digital object is used to open up a dialogue and create ongoing connections with those that are part of this engagement and so we see the digital object take on a specific role which focuses on fostering a relationship. This process of forming a relationship interlinks these projects to the notion of shared guardianship, as the digital object is a tool in which to bond participants and institutions together through open dialogue.

However, while these projects may appear to facilitate shared guardianship, Haidy Geismar identifies the problem of ownership around digital objects, with reference to the case of digital repatriation. Comparing it to Annette Weiner's paradox of 'keeping-while-giving', she argues that ownership is never truly distributed in these cases and instead it remains centralized (Weiner 1992; Geismar 2013: 257). In other words, the power dynamics do not change in these cases, and the digital museum object is offered as a pretence of giving whilst the authority 
over the museum object remains the same. Geismar's argument shows that ownership needs to be considered if shared guardianship is to take form. By sharing ownership, museums are truly distributing power across those that take part and give participants a stakeholder-like authority. In this respect, it is not enough to use a museum object as a way to prioritize a participant's experience; a material claim could also help to situate a participant's place as a stakeholder of that artefact.

In order to consider how a digital object could provide this material claim, it is important to return to the typical assumptions made about the digital object; that it lacks materiality and has an ephemeral quality. These assumptions are grounded in literature which refer to digital objects as having 'placelessness' and 'formlessness' or define digital objects as 'editable', 'open' and 'distributed' (Kallinikos et al. 2010; Odom et al. 2014). Indeed, the virtual has often been associated with 'the absence of existence' (Lévy 1998: 23). Paul Dourish, for instance, described how the virtual was traditionally viewed as 'the dissolution of the physical and its replacement with an ineffable digital abstraction' (Dourish 2016: 30). This assumption was also noted in this paper's case study where many of the participants noted the need for a material aspect in order to make an object feel 'more real' (Workshop 1, 2020; for an overview of the project's methodology, see below). Although literature indicates that this assumption is false (Blanchette 2011; Horst and Miller 2012; Shep 2015), this perception continues to persist and obstruct the potential use value of the digital museum object.

On the other hand, blockchain is viewed as a technology that creates value through its properties. Blockchain's materials build in authenticity to any token exchanged in its system as it documents every transaction that takes place, and this gives each token a provenance that is traceable within the infrastructure of blockchain. This makes it possible to see how blockchain could give a sense of permanence to digital objects, which in turn, gives these objects value. In this respect, blockchained digital objects have the possibility to be made into meaningful entities, an idea which, I would like to argue, could be used in the discussion of ownership and guardianship of museum objects. With blockchained digital objects, participants would gain an ownable material that can give them an authority as a stakeholder of that object. Therefore, the use of these blockchained digital objects would not aim to replace the physical; instead, these tokens might be used as a way to recognize shared guardianship and a person's role in the interpretation process of a museum object.

\section{Blockchain's Digital Materiality}

The main premise that can be drawn from the previous discussion is the understanding that blockchain could provide the 'material' claim needed in the formation of shared guardianship using digital museum objects. But how does blockchain's materiality differ from, say, a nonblockchain digital object's materiality? In what follows, Kirschenbaum's (2008) and Drucker's (2013) investigations on the subject will be used as a framework to explore the digital materiality of blockchain technology, and this discussion will highlight how the technology produces a material claim by reinforcing an authenticity, ownability, and an exchange value in digital objects traced in its network. This discussion aims to contextualize the use of blockchain in the case study noted in this paper by showing that blockchain adds a new layer of 'materiality' to tokenized museum objects that can inform and contribute to these objects' value and significance.

In brief, a blockchain is a distributed ledger system which enables the exchanging and ownership of digital tokens. The technology was first outlined in a white paper that was published in 2008 by the unknown collective or individual named Satoshi Nakamoto and this paper described blockchain as the digital infrastructure for the Bitcoin cryptocurrency. While Bitcoin is not the first digital currency to be made (Swartz 2018), this technology was considered revolutionary by its community because it addressed the fundamental challenge of the 'double-spending' problem when trying to send money digitally. This problem refers to the exchanging of the 'same' token more than once online, thus creating a copy of that token which reduces the 'original' token's ability to be exchanged for a value (Drescher 2017).

A blockchain addresses this issue by tracking each token anonymously on a public ledger, which is distributed across the nodes in the blockchain network in the 
form of blocks stacked in a linear chain (hence the name, blockchain). Therefore, this distributed public ledger holds every authenticated transaction that has taken place. The nodes can also act as miners, which are powerful computers backed by humans who compete against each other to solve mathematical algorithms, which unlock and authenticate the next block in the chain (Baldwin 2018). In other words, miners mine computational power to authenticate transactions so that they can be added to the public ledger. Therefore, these miners are like the 'authorities' in authenticating and maintaining the blockchain network, and they are incentivized to do this work with rewards in cryptocurrency for every block they mine (Calvão 2019).

The information kept in the blocks built into this distributed public ledger is encrypted using cryptography. This allows for private information to remain private whilst also allowing anyone to access the history of (pseudonymous) ownership (Nakamoto 2008). Therefore, blockchain is decentralized because, in theory, anyone can become a miner and help maintain the network. Blockchain is also distributed because its ledger is dispersed across the network of nodes, and it is open because anyone can access this public ledger.

Blockchain technology is now used far beyond cryptocurrencies, from tracing the provenance of physical diamonds and safeguarding government records (De Filippi and Wright 2018), to the buying and selling of digital art and creating a fairer system for music rights ownership (O'Dair 2019). In all of these cases, there is a need to trace the exchange of tokens using a blockchain and, in so doing, blockchain provides proof of authenticity, ownership, and an exchange value. For example, the National Archives collaborated with the University of Surrey to create ARCHANGEL, a project that aimed to bring trust to digital archives using blockchain technology

By applying cryptographic hashes to digital content in their archives, the researchers were able to produce a system that embedded an audit trail whilst also being distributed across a network of archives (Bell et al. 2018). Hence, the project promoted a collaborative attitude to archiving, which also helps to reinforce an authenticity in digital content. The company Verisart also offers an example of the potential use of blockchain in museums. Verisart is a platform where users can apply blockchain to a physical artwork, which creates a 'tamperproof certificate' that will document 'a museum quality record' of ownership for that piece. Such an idea could disrupt the traditional systems in place for documentation and facilitate the production of an immutable and digitized provenance for artworks in order to reduce the potential for forgeries and loss of documentation. Of course, this use case also brings its own challenges, namely the potential for human error or malicious behaviour in updating the documentation, sometimes referred to as the 'garbage in, garbage out' problem (Ito and O'Dair 2019).

However, in thinking about born-digital artwork, this problem is negated as the process of updating is done through the system of exchange. For example, KnownOrigin is a decentralized art gallery that offers a way to 'mint' digital artworks so that they are stored as a non-fungible token (also described as an NFT or a cryptocollectible). This process enables these digital artworks to be bought and sold as if they were exclusive forms of property because, once exchanged, new ownership is automatically recorded onto the blockchain through the practice of mining.

This process enables blockchain tokens, whether these are cryptocurrencies or NFTs, to have an exchange value embedded into them through the infrastructure and materials of the technology (Maurer et al. 2013; Bjerg 2016; Ferry 2016; Zimmer 2017). In the case of fungible tokens, such as the Bitcoin cryptocurrency, value is argued to be intrinsic to its materials and scholars ground this argument in the concept of 'digital metallism' and theories of commodity money (Ingham 2004: 212; Maurer et al. 2013). Specifically, 'digital metallism' argues that Bitcoin tokens also hold an intrinsic value based on its materials, which is justified by the 21 million Bitcoin coin cap that builds scarcity into the coins and a market fuelled by supply and demand (Maurer et al. 2013). This case is also made in relation to cryptocollectibles; the process of 'minting' a digital artwork individualizes it and gives it value through a digital scarcity that resembles the value given to a limited edition of a print (O'Dwyer 2018). However, it is important to note here that blockchain is not a digital rights management tool; it cannot stop others from copying and pasting a digital artwork. Therefore, the scarcity in NFTs produced 
through blockchain's materials is only a type of scarcity since NFTs do 'not reproduce the conditions of physical objects [...], it simulates the effects of these conditions' (Zeilinger 2018: 30). Thus, blockchain allows for the opportunity to claim ownership of a token and, through this act of claiming, the token gains an exchange value.

The arguments made here focus on blockchain's materiality as the source of its value. In defining digital materiality, Kirschenbaum (2008) considers two forms known as forensic and formal materiality. Forensic materiality refers to the individualization of traceable bits and this idea is also emphasized by Jean-François Blanchette when he describes the bits and bytes of the digital as 'material entities' (Blanchette 2011: 1042). Similarly, Sydney Shep defines this form of materiality in his description of digital materiality, which he notes as 'the palpable bits and bytes of electronic hardware and software [...], that leave traces, and that can be read as evidence [...] of these new communication forms' (Shep 2015: 322).

It is possible to see this individualization of bits and bytes in blockchain. Indeed, blockchain is made from blocks of data formed in a chain and these blocks of data are constructed from the bits and bytes that make each transaction. The traces of every transaction are recorded into the technology's infrastructure and so blockchain would not exist without these marks left by these exchanges. This notion relates to formal materiality, which describes the formal assemblage of these bits and bytes that construct the digital environment (Kirschenbaum 2008), in other words, formal materiality describes the symbolic forms of the digital; the digital object would not exist without this form. Hence, the blockchain token, such as a cryptocollectible, highlights this formal materiality and the traces of exchange gives this token a forensic materiality which can be used to prove authenticity and ownership.

As a result, blockchain's forensic materiality gives evidence of its formal materiality and this evidence also gives a blockchain token an authenticity that can be proven through the traces of bits documented in the infrastructure. By giving a token a proven authenticity, these tokens can be identified and singularized by their owners and this gives them an exchangeable value. Ole Bjerg's (2016) discussion on the similarities between Bitcoin tokens and credit money theory supports this idea of value creation through documentation. On a basic level, credit money involves an agreement between a creditor and a debtor which is then documented and so the value of this money stems from the recording of this agreement (Hart 2000). Bjerg (2016) argues that the value of a Bitcoin token also derives from this process of documentation as it gives it authenticity, and so the traces of bits and bytes that form blockchain's materiality also produces an exchangeable value in these tokens.

Blockchain's materiality might also be considered on a distributed and performative level. Drucker (2013) describes distributed materiality as the materiality formed through the network of entities a digital object depends on to exist. These could include servers, software and hosted environments. As a distributed and decentralized technology, blockchain's distributed materiality focuses on the network of nodes which each hold a copy of the ledger of transactions. This ledger is continually updated via this network and so all the traces of transactions that take place are documented across the system and it is through this documentation that authenticity is enforced, giving the blockchain token an exchange value.

This process of validating requires miners who mine the network to authenticate transactions before they are added to the distributed ledger and so there is also a performative element of materiality. Performative materiality considers 'the production of a work as an interpretative event' (Drucker 2013:para. 22). In other words, it acknowledges that actors are involved in the production of materiality. These actors do not have to be human, and in the case of blockchain, mining is carried out by powerful computers (backed by humans) who compete against each other to be the first to authenticate the transaction. Once authenticated, the transaction is added to each copy of the ledger by passing the information across the network (DuPont 2019). Therefore, the value of a blockchain token also depends on miners performing and executing the code to complete the transaction. Without this, blockchain tokens cannot exist.

Therefore, these discussions highlight how a blockchain's materiality depends on different forms, all of which contribute to a blockchain token's exchangeable value. On a forensic and formal level, the traces of the bits and bytes of a transaction are documented as part of the infrastructure of blockchain and this gives blockchain tokens an embedded value. 
This value is also reinforced through blockchain's distributed materiality as this process of documentation is distributed across the network. Of course, this all depends on a performative element provided by miners who authenticate the transactions and so the exchange value of a token is dependent on the network to perform and to produce a record which can be used to prove authenticity and ownership.

In this way, a blockchain's token's properties differ from that of a 'typical' digital object because its various elements of materiality provide it with an added value. In the context of museums, this added value could be used as a way to apply significance and meaning to digital museum objects. Indeed, these different elements of materiality give these tokens a sense of material claim because they become separate entities from the original digital collection; participants can claim ownership and the additional value of these tokens, which in turn, could produce a new meaningfulness and value. Therefore, the creation of exchange value through blockchain has the potential to produce other forms of value in the context of digital museum objects. The following examination of the NML case study aims to highlight to what extent this is the case.

\section{NML Case Study: Creating Value in Digital Museum Objects}

Between September 2018 and September 2020, the NML and the University of Manchester collaboratively worked on a project that explored how blockchain technology might be used to create meaningful digital museum objects. 'Meaningful' here refers to objects which have a value that connects a participant both to the physical museum object and to the museum itself. Hence 'meaningful' also relates to the notion of forging relations and value. The project recognized that this process of creating meaning would need to focus on creating personal value and personal connection for the participants involved and so the process took a collaborative approach to interpretation with the emphasis on looking at the perspectives of the participants towards certain objects. In so doing, the project also investigated how to challenge the curatorial authority of a museum and to reconceptualize the notion of ownership through the use of blockchain.

The project consisted of a three-part process where a group of seven participants were invited to a workshop at the Walker Art Gallery, an online exhibition was made based on the outcomes of this workshop, and cryptocollectibles were made based on the objects in this online exhibition. In this respect, the project was conceived with the idea that participants would gradually form a deeper connection to the objects by being part of this progression. The initial workshop took place in January 2020 and the participants took part in various activities, including discussing a personal possession that they brought to the workshop and exploring the collections of the Walker Art Gallery and World Museum so as to pick a museum object based on personal connection. Together, these possessions and museum objects formed an online exhibition called $\underline{\text { Crypto-Connections }}$ and would later be transformed into cryptocollectibles.

The decision to incorporate both personal possessions and museum objects was based on two reasons. Firstly, from the perspective of the exhibition, the personal possessions offered a more accessible way for visitors to consider the main premise of the exhibition. Secondly, from a data analysis view, the two types of objects offered different perspectives on whether blockchain creates meaningful digital objects. In the case of the personal possessions, the participants would already have a deep and meaningful connection to these objects and so these objects offered a way to explore to what extent a cryptocollectible could depict an established meaningful object. On the other hand, the museum objects represent a type of object where the project established the connection between the participant and the artefact and so these cryptocollectibles would highlight to what extent blockchain could represent the value created through the process of doing the project. In either case, the overall aim was to produce digital objects which felt ownable and authentic, and acted as a form of digital connection.

The cryptocollectibles of these objects were made using a digital decentralized gallery called the Possession Gallery, and this was produced specifically for the project by blockchain developers. This gallery is connected to the Ethereum blockchain and displays 
any cryptocollectibles made from its admin page. Figure 1 shows the box on the admin page which is used to create these cryptocollectibles. The cryptocollectible is given a title and description, which is copied from the online exhibition, and the URL link for the image on the online exhibition page is also embedded into the token and this is used as the main visual image. To complete the exchange, the participant needed to provide a digital wallet address. On clicking submit, the transaction would complete using Metamask, a commonly used decentralized app (Dapp) for web browsers that connects to blockchain. Once completed, the cryptocollectible would appear in the participant's wallet and it would also be displayed on the Possession Gallery (see fig.2). A second meeting with the participants took place in July 2020 in which they received their cryptocollectibles and could reflect on the process.

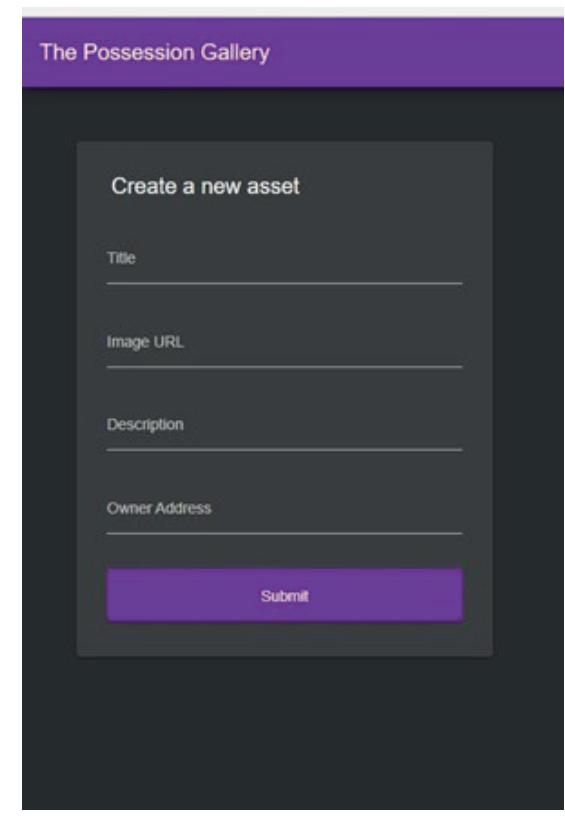

Figure 1: Box on admin page, The Possession Gallery, Liverpool Museums Website this is highlighted in a response from one participant to a question asking if blockchain would make a difference to their sense of ownership:

To me personally now, in this moment of time, no because blockchain means nothing to me now but sort of in ten years if you know I was used to hearing about it and had a better understanding of it and what it was all about then maybe.

(Participant C, Workshop 1, 2020)

In this respect, blockchain for many of these participants initially appeared to add nothing to digital objects and the potential for digital ownership. They still assumed the digital object to be this ephemeral, unownable entity.

Having created the cryptocollectibles, the second meeting offered the participants time to reflect on their assumptions and perceptions of blockchain and digital ownership and, initially, some of the participants struggled to comprehend their ownership of these cryptocollectibles. Moreover, while these participants recognized these digital tokens as a form of asset that could be translated into currency, they were still unsure of where this value is situated within their own objects. For example, one participant noted that their personal 


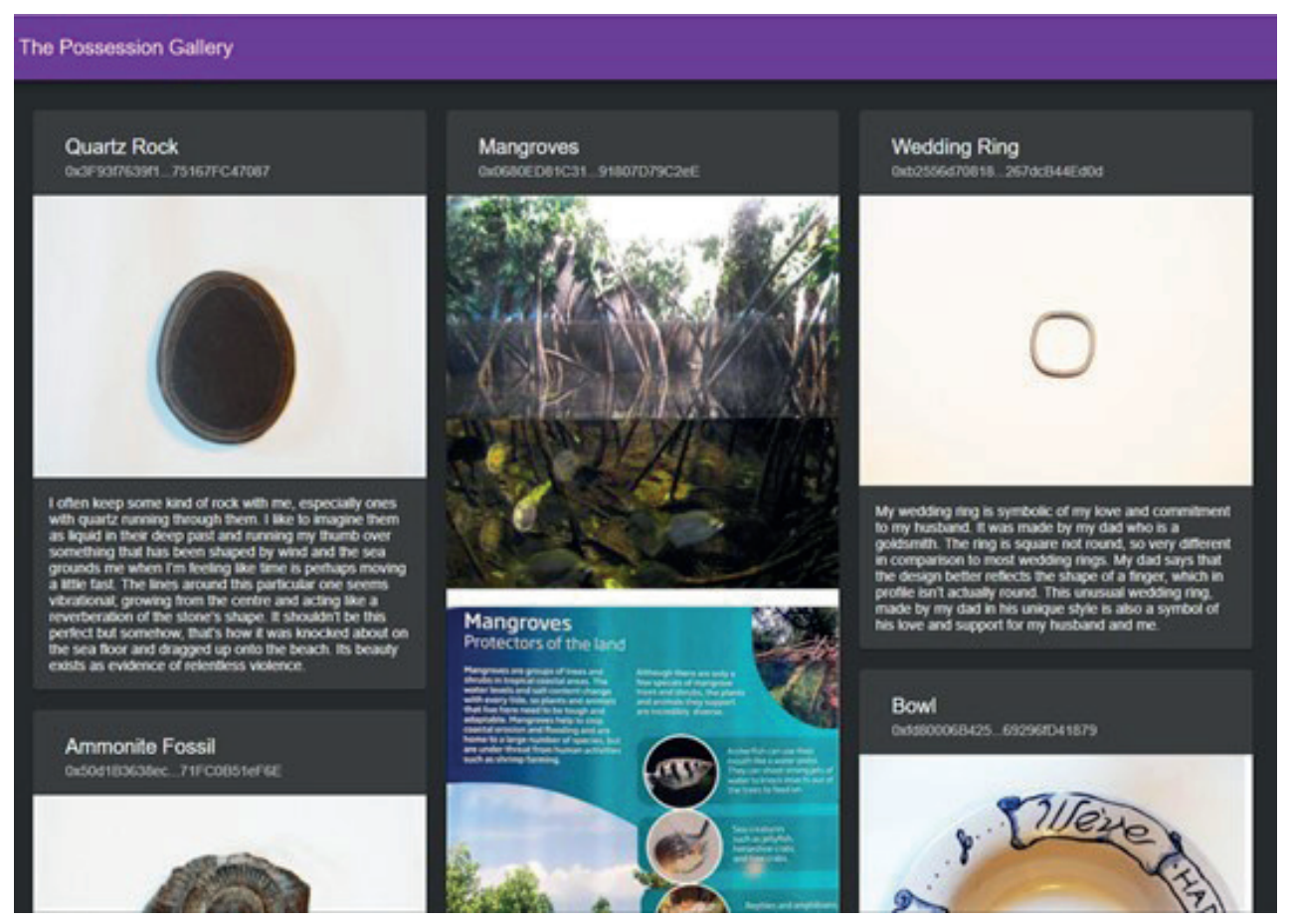

Figure 2: The Possession Gallery, Liverpool Museums Website.

possession had no monetary value; however, this process had added a value to it that they could not quite identify. In their words; 'I don't know where that [value] orbits around the actual object' (Participant B, Workshop 2, 2020). This comment suggests that some of these participants recognized that blockchain adds value, specifically an exchange value, but they were unsure of how that value is materialized or if that value connects to the physical object, and this response supports the idea that blockchain tokens hold a distinct value that can be used for exchange.

In thinking about this exchange value of the cryptocollectibles, it is salient to note that the process of creating these tokens was costly, as Ethereum carries a transaction fee which is used to carry out exchanges and execute cryptocollectibles. This transaction fee is also known as a 'gas price' and the money from this fee goes to whichever miner who authenticates that transaction as a reward. The process of rewarding miners was introduced as a way to encourage any node in the network to help to maintain the blockchain through mining transactions (Ethereum 2020). In theory, the miners are supposed to choose a 'gas price' which would be just enough to encourage them to carry out authentication; in practice, this means that it varies depending on the congestion in the network and the time of day. ${ }^{13}$

The 'gas price' was extremely high during the making of the cryptocollectibles, which various crypto news sources attribute to congestion caused by DeFi and stablescoins. ${ }^{14}$ This meant that the input of monetary value that 'makes' each cryptocollectible ranged from 40 to 60 gwei - the equivalent of $£ 4-£ 8,,^{15}$ - but this value is completely lost to the miners and to the upkeep of the blockchain. Therefore, this input of value is needed to materialize and authenticate the cryptocollectibles, which gives them a materiality supported by the network. Moreover, and as noted previously, this materiality gives blockchain tokens an exchange value because these tokens hold an authenticity which can be proven through the system. In this respect, blockchain's exchange value derives from an input of monetary value; however, this monetary value is disseminated into the network. 
As already noted, the participants recognized the exchange value in their cryptocollectibles, but this raises the question: would they choose to act upon this value? The assumption made in this project is that these blockchain tokens would be meaningful enough that the participants would not want to exchange their cryptocollectibles for cryptocurrency. This argument also resonates with the concept of the 'moral economy' which E.P. Thompson (1971) used to investigate the reasons behind the behaviour of people in the bread riots of eighteenth century England. The scholar argues that the rioters' behaviour can be explained through a sense of moral injustice caused by the modern capitalist economy challenging the traditional social community norms of that time (Thompson 1971). Martin Zeilinger (2013) also draws on this idea of the moral economy to describe the behaviour of chipmusic creators who challenge the restrictions of intellectual property rights online. Here, Zeilinger (2013) uses the moral economy to examine the differences between the open access digital culture and the restrictive intellectual property rights enforced online and, in so doing, demonstrates how chipmusic creators take a moralistic approach to the copying, creating and circulating of work online. These cases show how the moral economy is a useful framework to explain behaviour which advocates for a more moralist and community-based approach and, like in these examples, the moral economy can also be used to examine why the participants would choose to keep their cryptocollectibles.

In the second meeting, one participant remarked on how the museum object cryptocollectible is something they own, but it is also an imitation of something they do not own, as they describe: 'it's my own thing but it is a representation of something that is not owned by me' (Participant E, Workshop 2, 2020). This implies that there is confusion over the ownership as the blockchain token represents something that the museum owns but the token is owned by the participant. Building on this, it could be argued that the token reflects a point of intersection of ownership where both the museum and the participant are invested in the object, suggesting a shared or layered ownership forming. In terms of the moral economy, this indicates a moral obligation being established on the part of the participant as they may not feel it morally right to sell their cryptocollectible without the permission of the museum, even though they have full ownership of the cryptocollectible. This echoes the discussion on shared guardianship as the cryptocollectible effectively transforms the participant into a guardian of that digital object and, in so doing, the participant feels a duty to look after that object, implying that they should not sell it.

Moreover, it also seems unlikely that the participants would sell their possession cryptocollectibles as they represent a physical object that has personal value for them. This is supported by the participants' reflections on the value of owning a cryptocollectible. Notably, preservation was a key benefit for some of the participants. Participant A, who was the representative from the NML, pointed out that preservation is one of the main reasons to digitize an object in the first place and a cryptocollectible adds an enhancement to this process as it is something that the participant exclusively owns. This participant also recalled how they had momentarily lost their personal possession, and during this time, they had thought that the cryptocollectible could at least be a reminder of the lost possession. In this way, the cryptocollectible was a form of compensation for the potentially lost item; it preserved the object digitally and exclusively in the participant's wallet.

Participant $F$ made a similar argument when they commented on how the cryptocollectible provides context for the digital image. If something happened to the participant or they lost the physical object, the cryptocollectible offers a permanent digital representation of their memory and connection to that item. The participant noted how this process of materialization and preservation of the memory is probably more valuable than the preservation of the physical object, and so the cryptocollectible provides a way of creating an exclusively owned and preserved digital memory. It is clear from these reflections that many of the participants saw the value in the cryptocollectible for its ability to preserve the object and their memory and this connection to personal memory makes it seem unlikely that the participant would want to sell their memory for money. As a result, there is a clear moral argument at work which encourages the participants to keep their cryptocollectibles rather than sell them for a monetary value.

These arguments could also be used for the museum object cryptocollectibles, as these also personalize and contextualize the museum objects for the participants. In this 
respect, these cryptocollectibles are made meaningful through two aspects: firstly, as a moral keepsake, since there is an intersection of ownership over the museum object cryptocollectible and this implies that the cryptocollectible could represent a connection to the museum in the form of shared guardianship. Secondly, the cryptocollectibles are a materialization of personal experiences and memories and so they hold a personalization which gives them personal value, an idea that is supported by the fact that digital memory was a prominent theme used to describe the cryptocollectibles throughout the second meeting.

The ideas discussed here look at how blockchain tokens reimagine the use of the digital museum object as a potential ownable digital memory and this idea relates to the earlier discussion on Meehan's (2020) argument and the role of the digital object as a memory meeting point. However, in the case of this project, there are multiple digital objects which represent the different memories, and these are exclusively owned by the memory owners. This notion also connects to Keith Hart's (2000) description of money as a form of memory token. He argues that the value of money is not intrinsic to the materials; instead, it derives from the act of remembering exchanges within a community. In this way, money is like an instrument for collective memory and a form of memory bank in which its value derives from the process of recording (Hart 2000). Indeed, even the word money derives from Moneta, the Latin equivalent of the Greek goddess of memory called Mnemosyne (Hart 2000).

Of course, a blockchain token embodies this understanding since every point of exchange is documented into the infrastructure of the technology. But these cryptocollectibles also extends this notion of a token of memory as the act of exchange between the museum and the participant permanently documented the participant's personal experience to that specific digital version of the object. This process also fractures the digital collection into multiple and digitized perspectives and The Possession Gallery in this case study acts as a memory meeting point, because any cryptocollectible made from its admin page is also depicted in this decentralized gallery. Hence, this space helps to centralize these different memories to form a collective memory space.

The potential with this space is to continue to build on the different ways of looking at an object where the memories are presented together in this gallery. In the second meeting, one participant compared this idea to the contributions to a Wikipedia page where the 'wisdom of the crowds' is used to build the knowledge about a topic (Surowiecki 2005). This suggests that the cryptocollectibles could be a collection of interpretations about an object, in which each contributor gains their own digital entity to signify their role in the process. In owning these digital entities, these participants can gain a collection of their personal memories and this can help to bind the participant to both the collection and the museum. Hence, these cryptocollectibles could prove to be a way of making meaningful connections between contributors and a cultural institution, which in turn would also create a whole new digital collection represented in the decentralized gallery that embodies these different perspectives.

\section{Conclusion}

In drawing some conclusions from this analysis, we can see that blockchain's infrastructure impacts the concept of value in digital objects. This value derives from the process of documenting and investing in the blockchain. For example, in the case study the digital tokens were created through paying the 'gas price' to the miners, who then authenticated these tokens by recording the process of exchange into the Ethereum blockchain. In so doing, the tokens gain an exchange value as they are made exclusive, and the participants could claim ownership over these tokens.

However, although this exchange value is important, this project also highlights a different forming of value, one that is more personal and meaningful in nature. In this instance, it is not about simply investing money to create authenticity; the participants have also invested their own personal experience into the project so as to produce a sense of connection and potential guardianship. We can see this in the case of the museum object cryptocollectibles, where there is this layered ownership forms in which both the participant and the museum hold a stake in what the cryptocollectible represents. Hence, prioritizing the participants' experience in this project enabled them to feel invested in the cryptocollectible, which in turn has the potential to create personal value and shared guardianship. 
In this way, investment is twofold, where the museum invests to produce authentic and ownable digital commodities, and the participant invests part of themselves into the collection. This process also highlights blockchain's potential to reconceptualize the notion of ownership over digital museum collections. The technology digitally fragments the collection into ownable pieces in which these portions help to bind the participant, object and museum. This binding together forms a collective ownership, an idea that resonates with discussions on open access policy in GLAM institutions, where scholars propose that digital collections should be free and available to download and be used by online audiences ( Sanderhoff 2013, 2014; Tanner 2016). ${ }^{16}$ These arguments are founded on the idea that cultural heritage is 'common property' and 'belongs to us all' (Sanderhoff 2014: 9), and a similar point was made by participant $A$ about the project during the second workshop: 'it goes back to that idea that it's a national museum therefore its owned by the nation' (Participant A, Workshop 2, 2020); in other words, national collections invoke a sense of collective ownership, they are in a way 'common property'. While the open access discussion argues for cultural institutions to reconsider their approach to the rights of their digital collections so that they foster availability and reuse, whereas I have argued for the use of blockchain to develop a new and different form of collective ownership, in fact, both forms could exist in relation to one another. As such, this coexistence extends the argument and identifies how blockchain has the potential to challenge our understanding of ownership in the digital museum space.

Of course, there are a number of problems to recognize in this use of blockchain. Firstly, the discussion on ownership over the museum cryptocollectible suggests that the process can form shared guardianship. However, such projects risks the 'keeping-while-giving' problem, if participants are not given the full opportunity to use their token as they see fit. Hence, these tokens should be made into embodiment of shared authority as well as shared ownership. In other words, these tokens need to have some form of utility value other than simply holding an exchange value. This could be in the form of displaying these cryptocollectibles with the use of digital frames such as TokenCast, ${ }^{17}$ or these tokens could relate to a governance model where owners can be part of the decision making about a particular object, and this idea could be particularly salient in the context of objects with historical or cultural value. Secondly, and as noted earlier, the NML project faced high 'gas fees' during the project and this points to a limitation of wider blockchain use, namely the problem of scalability, and this will continue to be a problem until the blockchain community finds a permanent solution.18 Public understanding and engagement of blockchain is also a challenge and this can lead to practical issues. For example, in this project, The Possession Gallery can only be accessed via a digital wallet, meaning that visitors must either have, or know how to create, a digital wallet before being able to access this platform, and so the application of blockchain builds an immediate digital barrier to many visitors.

Nevertheless, this discussion on blockchain in the context of the museum has shown how the technology can build on conversations around ownership in the digital museum space. In drawing on how blockchain produces a form of property in digital objects, the digital museum object takes on a new role in which it acts as its own entity that also binds people together. In this way, the digital museum collection can become a new set of tools for forging relations and connections between people, objects, and the museum.

Received: 15 April 2020

Finally accepted: 1 June 2021

\section{Notes:}

1 See Nakamoto, S. (2008) 'Bitcoin: A peer-to peer electronic cash system', Satoshi Nakamoto Institute. Available at: https://nakamotoinstitute.org/bitcoin/ (Accessed: 10 December 2018).

2 See Ethereum (2020) Ethereum Whitepaper. Ethereum. Available at: https:// ethereum.org/whitepaperl (Accessed: 6 May 2020).

$3 \quad$ Not all blockchains are public; however, the project discussed in this article works on a public blockchain named Ethereum. 
Also see George, C. (2020) 'Diamond Provenance at Every Link of the Value Chain', Everledger, 1 May. Available at: https://www.everledger.io/diamond-provenance-atevery-link-of-the-value-chain/ (Accessed: 15 July 2020).

Also see Monegraph (2018) Monegraph. Available at: https://monegraph.com/home (Accessed: 1 November 2018).

See ARCHANGEL (2020) Surrey Blockchain | ARCHANGEL Project, Surrey Blockchain. Available at: https://blockchain.surrey.ac.uk/projects/archangel.html (Accessed: 23 October 2020).

See Verisart (2020) Verisart. Available at: https://verisart.com/ (Accessed: 23 October 2020).

See KnownOrigin (2020) KnownOrigin.io, KnownOrigin. Available at: https://dapp. knownorigin.io (Accessed: 29 January 2020).

https://www.liverpoolmuseums.org.uk/collections/cryptoconnections, accessed 22 July 2020.

https://collectibles.liverpoolmuseums.org.uk/\#l, accessed 22 July 2020.

https://metamask.io/, accessed 25 July 2020.

See Design Informatics (2019) Block Exchange. Edinburgh: University of Edinburgh \& Northumbria University. Available at: http://blockexchange.designinformatics.org/ wp-content/uploads/2017/01/DesignInformatics_BlockExchange.pdf (Accessed: 21 March 2019).

See Ethgasstation.info (2020) ETH Gas Station | FAQ, Eth Gas Station. Available at: https://ethgasstation.info/FAQpage.php (Accessed: 27 July 2020)

Stablescoins are a form of cryptocurrency which is used to provide stability in price (Lloyd, 2019). Defi is a protocol that builds on the Ethereum network - https:// defiprime.com/, accessed 27 July 2020.. See Young, M. (2020) Ethereum Gas Usage Tops All-Time High Amid DeFi and Stablecoin Tear, BelnCrypto. Available at: https://beincrypto.com/ethereum-gas-usage-tops-all-time-high-amid-defi-andstablecoin-tear/ (Accessed: 27 July 2020, Lloyd, J. (2019) What is a Stablecoin and How do They Work?, BelnCrypto. Available at: https://beincrypto.com/learn/ what-is-stablecoin/ (Accessed: 27 July 2020). Chong, N. (2020) 'Ethereum Fees Just Hit a Multi-Year High, But There Are Solutions', NewsBTC, 29 June. Available at: https://www.newsbtc.com/2020/06/29/ethereum-fees-multi-year-high-solutions/ (Accessed: 27 July 2020). Baker, P., Keoun, B. and Godbole, O. (2020) 'First Mover: Ethereum a Victim of Its Own Success as Fees Soar, Vitalik Complains', CoinDesk, 23 July. Available at: https://www.coindesk.com/first-mover-ethereum-a-victim-ofits-own-success-as-fees-soar-vitalik-complains (Accessed: 27 July 2020).

Gwei is a unit of Ether, the cryptocurrency for the Ethereum blockchain; there are $1,000,000,000$ gwei in one Ether (Ethgasstation.info, 2019). The gas price noted here is extremely high in contrast to previous gas prices; for example, the gas price on the day of the workshop in January averaged 7.5 gwei, whilst the gas price on the day of the second meeting was 40 gwei (etherscan.io, 2020). (Ethgasstation. info (2019) 'What is Gwei?', ETH Gas Station. Available at: https://ethgasstation. info/blog/gwei/ (Accessed: 30 July 2020), and etherscan.io (2020) Ethereum Average Gas Price Chart, Ethereum (ETH) Blockchain Explorer. Available at: http:// etherscan.io/chart/gasprice (Accessed: 28 July 2020). 
$16 \quad$ Also see Kelly, K. (2013) Images of Works of Art in Museum Collections: The experience of open access: a study of 11 museums. Available at: https://www.clir. org/pubs/reports/pub157/pub157.pdf (Accessed: 22 November 2018).

17 See https://tokencast.net/, accessed 29 October 2020.

18 The community are currently looking at 'off-chain' solutions such as the Plasma project and the Raiden network (EthHub, 2020; Raiden Network, 2020). See Raiden Network (2020) What is Raiden?, Raiden Network. Available at: https:/l docs.raiden.network/ (Accessed: 29 October 2020), and EthHub (2020) Plasma - EthHub, EthHub. Available at: https://docs.ethhub.io/ethereum-roadmap/layer-2scaling/plasma/ (Accessed: 29 October 2020).

\section{References}

Atasoy, O. and Morewedge, C.K. (2018) 'Digital Goods Are Valued Less Than Physical Goods', Journal of Consumer Research, 44 (6) 1343-57. doi: 10.1093/jcr/ucx102.

Baldwin, J. (2018) 'In Digital We Trust: Bitcoin Discourse, Digital Currencies, and Decentralized Network Fetishism', Palgrave Communications, 4 (1) 1-10. doi: 10.1057/s41599-018-0065-0.

Bell, M. et al. (2018) 'Using blockchain to engender trust in public digital archives', in Proceedings iPRES 2018. International Conference on Digital Preservation, retrieved from OSF, doi: 10.17605/OSF.IO/KEFJ8.

Bjerg, O. (2016) 'How is Bitcoin Money?', Theory, Culture \& Society, 33 (1) 53-72. doi: 10.1177/0263276415619015.

Blanchette, J.-F. (2011) 'A Material History of Bits', Journal of the American Society for Information Science and Technology, 62 (6) 1042-57. doi: 10.1002/asi.21542.

Brown, D. (2007) 'Te Ahua Hiko: Digital Cultural Heritage and Indigenous Objects, People, and Environments', in Fiona Cameron and Sarah Kenderdine (eds) Theorizing Digital Cultural Heritage, 77-91, Cambridge, Mass.: MIT Press.

Calvão, F. (2019) 'Crypto-miners: Digital Labor and the Power of Blockchain Technology', Economic Anthropology, 6 (1) 123-34. doi: 10.1002/sea2.12136.

Cameron, F. and Robinson, H. (2007) 'Digital Knowledgescapes: Cultural, Theoretical, Practical and Usage Issues Facing Museum Collection Databases in a Digital Epoch', in Fiona Cameron and Sarah Kenderdine (eds) Theorizing Digital Heritage, 165-89, Cambridge, Mass.: MIT Press.

De Filippi, P. and Wright, A. (2018) Blockchain and the Law, Cambridge, Mass. \& London: Harvard University Press.

Dourish, P. (2016) 'Rematerializing the Platform: Emulation and the digital-material', in Sarah Pink, Elisendra Ardèvol and Débora Lanzeni (eds) Digital Materialities: Design and Anthropology, 29-44, London \& New York: Bloomsbury Publishing.

Drescher, D. (2017) Blockchain Basics: A Non-Technical Introduction in 25 Steps, New York, Apress.

Drucker, J. (2013) 'Performative Materiality and Theoretical Approaches to Interface', Digital Humanities Quarterly, 007 (1). http://digitalhumanities.org/dhq/ vol/7/1/000143/000143.html. 
DuPont, Q. (2019) Cryptocurrencies and Blockchains, Cambridge \& Malden: Polity Press.

Ferry, E. (2016) 'On Not Being a Sign: Gold's Semiotic Claims', Signs and Society, 4 (1) 57-79. doi: 10.1086/685055.

Geismar, H. (2008) 'Cultural Property, Museums, and the Pacific: Reframing the debates', International Journal of Cultural Property, 15 (2) 109-22. doi: 10.1017/ S0940739108080089.

(2013) 'Defining the Digital', Museum Anthropology Review, 7 (1-2) 254-63.

(2018) Museum Object Lessons for the Digital Age, London: UCL Press.

Hart, K. (2000) The Memory Bank, London: Profile Books.

Hazan, S. (2007) 'A Crisis of Authority', in Fiona Cameron and Sarah Kenderdine (eds) Theorizing Digital Cultural Heritage,133-48, Cambridge, Mass. \& London: MIT Press.

Hogsden, C. and Poulter, E.K. (2012) 'The Real Other? Museum Objects in Digital Contact Networks', Journal of Material Culture, 17 (3) 265-86. doi: $10.1177 / 1359183512453809$.

Hooper-Greenhill, E. (2000a) Museums and the Interpretation of Visual Culture, London \& New York: Routledge.

(2000b) 'Changing Values in the Art Museum: Rethinking Communication and Learning', International Journal of Heritage Studies, 6 (1) 9-31. doi: $10.1080 / 135272500363715$.

Horst, H.A. and Miller, D. (2012) Digital Anthropology, English ed., London: Berg.

Ingham, G. (2004) 'The Emergence of Capitalist Credit Money', in L. Randall Wray (ed) Credit and State Theories of Money, 173-222, Cheltenham, UK: Edward Elgar.

Ito, K. and O'Dair, M. (2019) 'A Critical Examination of the Application of Blockchain Technology to Intellectual Property Management', in Horst Treiblmaier and Roman Beck (eds) Business Transformation through Blockchain: Volume II, 317-35, Cham: Springer International Publishing. doi: 10.1007/978-3-319-99058-3_12.

Jeffrey, S. (2015) 'Challenging Heritage Visualisation: Beauty, Aura and Emocratisation', Open Archaeology, 1 (1) 144-52. doi: 10.1515/opar-2015-0008.

Jeffrey, S., Jones, S., Maxwell, M., Hale, A. and Jones, C. (2020) '3D visualisation, Communities and the Production of Significance', International Journal of Heritage Studies, 26 (9) 885-900. doi: 10.1080/13527258.2020.1731703.

Kallinikos, J., Aaltonen, A. and Marton, A. (2010) 'A Theory of Digital Objects', First Monday, 15 (6). doi: 10.5210/fm.v15i6.3033.

Kirschenbaum, M. (2008) Mechanisms, Cambridge, Mass. \& London: MIT Press.

Lévy, P. (1998) Becoming Virtual: Reality in the Digital Age, New York: Plenum.

Marstine, J. (2011) 'The Contingent Nature of the New Museum Ethics', in Janet Marstine (ed) The Routledge Companion to Museum Ethics: Redefining Ethics for the Twenty-First Century Museum, 3-25, Florence, USA: Routledge. 
(2017) Critical Practice: Artists, Museums, Ethics, London \& New York: Routledge, Taylor \& Francis Group.

Maurer, B., Nelms, T.C. and Swartz, L. (2013) '"When Perhaps the Real Problem is Money Itself!": The Practical Materiality of Bitcoin', Social Semiotics, 23 (2) 261-77. doi: 10.1080/10350330.2013.777594.

Meehan, N. (2020) 'Digital Museum Objects and Memory: Postdigital Materiality, Aura and Value', Curator: The Museum Journal. doi: 10.1111/cura.12361.

Merriman, N. (2015) 'The Future of Collecting in "Disciplinary" Museums: Interpretive, Thematic, Relational', in Macdonald, S \& Leahy, H. (Eds.) The International Handbooks of Museum Studies, 249-64, Chichester, John Wiley \& Sons. doi: 10.1002/9781118829059.wbihms211.

Ngata, W., Ngata-Gibson, H. and Salmond, A. (2012) 'Te Ataakura: Digital Taonga and Cultural Innovation', Journal of Material Culture, Amiria Salmond and Billie Lythberg (eds) 17 (3) 229-44. doi: 10.1177/1359183512453807.

O'Dair, M. (2019) Distributed Creativity: How Blockchain Technology will Transform the Creative Economy, Cham: Palgrave Macmillan.

Odom, W., Zimmerman, J. and Forlizzi, J. (2014) 'Placelessness, Spacelessness, and Formlessness: Experiential Qualities of Virtual Possessions', in Proceedings of the 2014 Conference on Designing Interactive Systems - DIS '14. the 2014 conference, 985-994, Vancouver: ACM Press. doi: 10.1145/2598510.2598577.

O'Dwyer, R. (2018) 'Limited Edition: Producing Artificial Scarcity for Digital Art on the Blockchain and its Implications for the Cultural Industries', Convergence, 1-21. 26 (4) doi: 10.1177/1354856518795097.

Sanderhoff, M. (2013) 'Open Images. Risk or Opportunity for Art Collections in the Digital Age?', Nordisk Museologi, 0 (2) 131-46. doi: 10.5617/nm.3083.

(2014) Sharing is Caring: Opening and Sharing in the Cultural Sector, Copenhagen: Statens Museum for Kunst.

Shep, S.J. (2015) 'Digital Materiality', in Susan Schreibman, Ray Siemens and John Unsworth (eds) A New Companion to Digital Humanities, 322-30, Chichester: John Wiley \& Sons, Ltd. doi: 10.1002/9781118680605.ch22.

Speed, C. (2012) 'Mobile Ouija Boards', in Elisa Giaccardi (ed) Heritage and Social Media, 179-96, London \& New York: Routledge.

Srinivasan, R., Becvar, K.M., Boast, R. and Enote, J. (2010) 'Diverse Knowledges and Contact Zones within the Digital Museum', Science, Technology, \& Human Values, 35 (5) 735-68. doi: 10.1177/0162243909357755.

Surowiecki, J. (2005) The Wisdom of Crowds: Why the Many are Smarter Than the Few, London: Abacus.

Swartz, L. (2018) 'What was Bitcoin, What will it Be? The Techno-economic Imaginaries of a New Money Technology', Cultural Studies, 32 (4)623-50. doi: 10.1080/09502386.2017.1416420.

Tanner, S. (2016) 'OpenGLAM: The Rewards (and Some Risks) of Digital Sharing for the Public Good', in Andrea Wallace and Ronan Deazley (eds) Display At Your Own Risk. Available at: https://displayatyourownrisk.org/tanner/ (Accessed: 23 February 2019). 
Thompson, E.P. (1971) 'The Moral Economy of The English Crowd in The Eighteenth Century', Past \& Present, 50 (1) 76-136. doi: 10.1093/past/50.1.76.

Walsh, P. (1997) 'The Web and the Unassailable Voice', Archives and Museum Informatics, 11 (2) 77-87.

Weiner, A. (1992) Inalienable Possessions: The Paradox of Keeping-While Giving, Berkeley and Los Angeles: University of California Press.

Zeilinger, M. (2018) 'Digital Art as "Monetised Graphics”: Enforcing Intellectual Property on the Blockchain', Philosophy \& Technology, 31 (1) 15-41. doi: 10.1007/s13347-0160243-1.

Zeilinger, M.J. (2013) 'Chiptuning Intellectual Property: Digital Culture Between Creative Commons and Moral Economy', IASPM Journal, 3 (1) 19-34.

Zimmer, Z. (2017) 'Bitcoin and Potosí Silver: Historical Perspectives on Cryptocurrency', Technology and Culture, 58 (2) 307-34. doi: 10.1353/tech.2017.0038.

\section{Author}

Frances Liddell

frances.liddell@postgrad.manchester.ac.uk

ORCID iD: https://orcid.org/0000-0001-9645-3845

University of Manchester

United Kingdom 